\title{
Graphical Modeling and simulation of the Hydropower Plant Switch
}

\author{
Yihui Zhou ${ }^{1}$, Tangxian Chen ${ }^{1}$ and Binqiao Zhang, ${ }^{2}$ \\ ${ }^{1}$ College of Electrical and New Energy, China Three Gorges University, Yichang, China \\ ${ }^{2}$ School of Hydropower and Information Engineering, Huazhong University of Science and \\ Technology, Wuhan, China
}

Keywords: hydropower plant simulation, switch, topology analysis, Graphical Modeling.

\begin{abstract}
The circuit breaker and the relevant disconnecting switches of the hydropower plant have different varieties in large quantity. Due to the different specific operation processes of the switches, the simulation is quite complex. Adopting the graphical and modular modeling approach, classify the switch system into various sub-modules to set up the universal models, and put forward the new methods of the logical interlock judgment for the switch action process on the basis of the connecting line merging topology analysis algorithm, improving the generality of the modeling and simulation of the electrical switch system. The modeling method provides an effective way for the large-scale customization of the hydropower plant simulation system.
\end{abstract}

\section{Introduction}

Guaranteeing the safe, stable and economical operation of the hydropower plant is the top priority of the hydropower production. The safe and practical operation training and analytical research platform plays an important role in enhancing the analytical research and operation ability of the hydropower employees. The hydropower plant simulation system is the computer simulation software facing "the whole station, the whole scope and the whole process" of plant [1], which can simulate various complex working conditions so that the operators can completes the analysis and simulation operation fully and effectively within a short time. With a large number of hydropower plants putting into operation successively, the simulation system has extensive and urgent demands.

Hydropower plant is a complex large-scale system for the tight coupling of hydraulic, mechanical, electric devices or processes [2].As an important part of the hydropower plant simulation system, the simulation model of the electrical switch devices and system is the central link between the operations under the normal, abnormal, emergency accident working conditions, and has been widely applied in the electrical switching operation, the startup-shutdown of generator unit and the action of relay protection device. A set of reliability, real-time and general simulation model of electric switch equipment is the key to the hydropower plant simulation system.

Because of the differences in the type of generator unit, main wiring form and operating standard between each hydropower plant, the traditional electrical switch simulation modeling focuses on the specified hydropower plant and their specific requirement to conduct the customized development with long period and high cost, which is difficult to adapt to the urgent demand of the large-scale hydropower simulation [3]. With the simulation model of the electrical switch system of the hydropower plant as the target, this paper researches the universal graphical modeling and simulation approach to lay the foundation for large-scale customization of hydropower plant simulation system.

\section{Electrical Switch System Function Modeling of the Hydropower Plant}

The switch operation is an important and frequent business during the daily production of the hydropower plant. It is quite complex for the variety and operating modes of the switch. Take the switch operation of Longtan Hydropower plant as the example, it includes the switch operation of $500 \mathrm{kV}$ GIS, $18 \mathrm{kV}$ generator outlet, $10 \mathrm{kV}$ auxiliary power system and $400 \mathrm{~V}$ auxiliary power system. Not only does the switch operation can be achieved through the remote operation on the upper 
computer monitoring system, but also it can be achieved in the local control unit. This leads to the high complexity of the switch operation, which brings difficulties to the generality of the modeling.

The switch operation can be classified into the basic operation and switching operation. The basic operations include the switch operations of the circuit breaker, the isolating switch and the earthing switch. And the switching operation mainly refers to the on-off operation of the electrical equipment, which is achieved by such way as the basic operations of the switch are combined according to the regulated operating sequence. The function modeling and simulation of the switch system can be classified into the basic operation simulation and the switching operation simulation.

The switch operation needs to meet some conditions. When the remote automatic operation is conducted, the judgment of the conditions is achieved through the automatic control process of the switch operation while for the local operation, the operators judge whether the conditions are up to the standard manually according to the requirements of the operation specification and the relevant interlock conditions of the equipment itself. The simulation of the switch automatic control process and the simulation of the operation specification is the important content of the switch system modeling.

In the operation of the switch equipment, the human computer interface sends the right control command to the local control unit (LCU) first. Then the LCU equipment conducts the local interlock judgment, namely, the judgment of the five-prevention regulations of the electrical system according to the pre-completed program. If the operation is legal, send the command to the switch cabinet, or the alarming window will pop in the human computer interface, displaying the detailed information on the cause of the operation failure and something else. After receiving the operating command, the switch cabinet checks the failure. If the check result is that there is no fault in the current switch hardware structure, the switch will work normally and the state of the switch changes correspondingly. Otherwise, the corresponding fault phenomenon appear (for instance, the fault indicator light turns on) and submit the detailed information on fault to the human computer interface [4]. Fig. 1 shows the overall operating process of the switch.

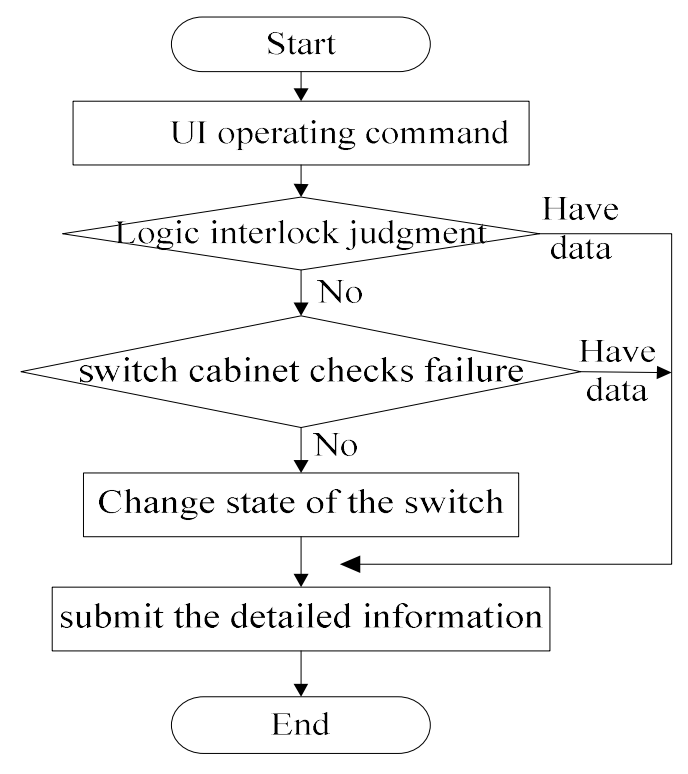

Figure 1. Control Process of the Switch Operation.

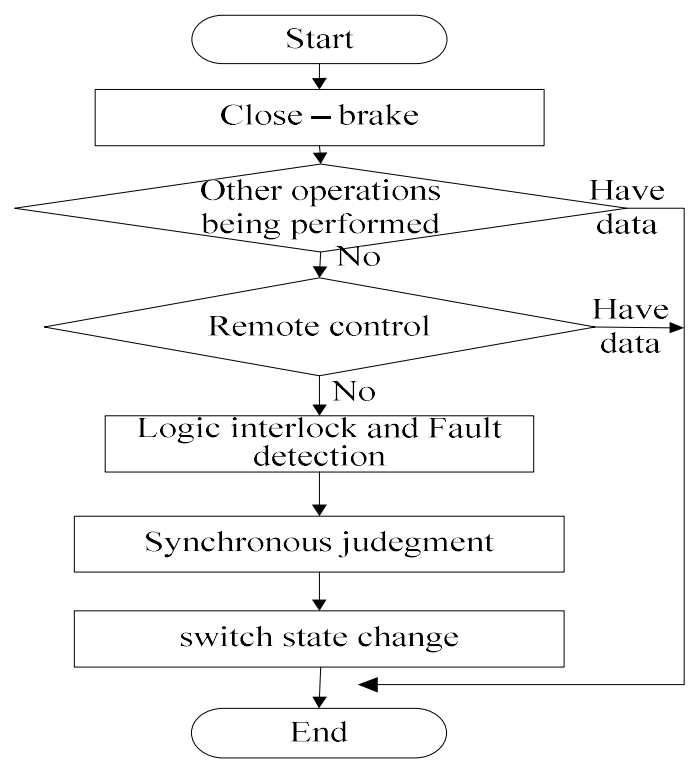

Figure 2. Switching-on Modular Process

\section{Graphical Modeling of the Switch system}

Hierarchy Modular Modeling of the Basic Operation. As the switches of the hydropower plant have quite a few varieties in large quantity and every switch is different in the specific motion process, if modeling is conducted for each one, the workload of the programming will be quite heavy and the model lacks in the generality, not available to be transplanted to the electrical switch system 
simulation of the other hydropower plants conveniently. In order to achieve the generality of the switch simulation model, adopt the graphical and modular modeling approach. According to the function and position of the electrical switch system in the hydropower plant, classify it into several great modules and in turn, every module includes several sub-modules that can be divided into smaller ones specifically according to the needs. It is easy to set up universal enough simulation model for the sub-modules and then conduct the modular combination for the sub-models to establish the complete switch system with the graphical method [5].

Take the modular modeling of the remote and synchronous switching-on of the circuit breaker in the switch station as the example to introduce the modular modeling approach of the whole switch system. Firstly, the same circuit breaker of the switch station has three operating modes including synchronous switching-on, non-pressure switching-on and switch separating. Only one user can conduct one operation at the same time. In this way, the interlock for the other operations of the same circuit breaker shall be considered in the switch operation, which can be modeled as a universal sub-module. Then, during the electrical switch operation process in the hydropower plant, conduct the judgment for the state and control modes of the circuit breaker. And the control modes can be modeled as the universal modules as well. Based on the similar classification method and referring to the overall framework of the control process of the switch operation in Fig. 1, provide the synchronous switching-on modular process diagram of the circuit breaker in the switch station in Fig. 2.

Graphical Modeling of the Switch Interlock Logic. Although the electrical switches of the hydropower plant have quite a few varieties in large quantity, after the hierarchy modular processing, the rest important function for both the circuit breaker equipment and the disconnecting switch equipment is the judgment for the interlock relationship to avoid the mid-operation. During the switching operation process of the equipment, the operators must abide by a certain operating sequence strictly. For example, whether Equipment A can be operated depends on the state of the equipment related to Equipment A. Only when the relevant equipment is under right state can the Equipment $\mathrm{A}$ be operated. The relation between the equipment to be operated and the relevant equipment is presented by the logical expression, the logical value of which determines the manipuility of Equipment A. And this expression is called as the logical interlock function of Equipment A. Before operating the equipment, only calculate the logical interlock function of this equipment and judge the validity of the operation according to the result without the needs of searching and matching of the rules, which improves the inference and judgment speed obviously. However, it is needed to set up the corresponding interlock relationship mapping table to know the equipment that is established the interlock relation with Equipment A accurately, namely the equipment that is the members of the logical interlock function expression. Table 1 is the interlock relationship information table that the 5011 switching-on of the circuit breaker in the main electrical connection shown in Fig. 3.

Table 1. Switching On-Off Logical Interlock Relationship

\begin{tabular}{|c|c|c|c|}
\hline Equipment & Operation & Interlock switch on & Interlock switch off \\
\hline 5011 & On & 50111,50112 & 501117,501127 \\
\hline 50112 & On & 501127 & 5011 \\
\hline
\end{tabular}
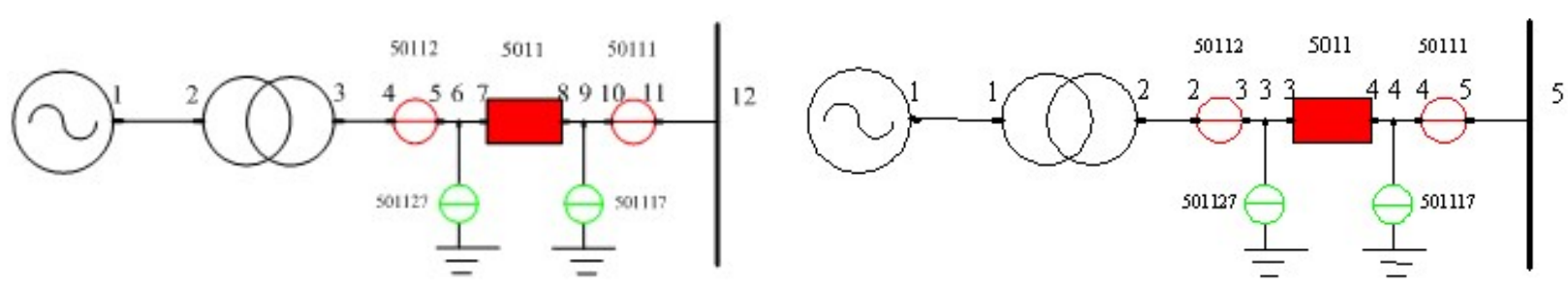

Figure 3. the Initial Port Number

Figure 4. the Port Number after the Emerging of Connecting Lines 
It is troublesome to set up such switch interlock information table manually. What's worse, once the main connection shown in Fig. 3 changes, it is needed to modify the interlock relationship information table manually, resulting in the generality shortage of the electrical switch model. In order to make the electrical switch model provided with the excellent self-adaption and generality, modify the interlock relationship information table of the switch operation process automatically according to the change of the main electrical connection. Here, adopt the topology analysis algorithm of "merging of the connecting line" to set up the information table of logical interlock function members of the switch operation process automatically.

First of all, draw the pixels of the circuit breaker, disconnecting switch and earthing switch, and the defined attributes are as follows: (1) Circuit breaker: port quantity, name of port 1, name of port 2, belonging unit and voltage classes; (2) Disconnecting switch: port quantity, name of port 1, name of port 2, belonging unit and voltage classes; (3) Earthing switch: port quantity, port name, belonging unit and voltage classes; (4) Connecting line: port quantity, name of port 1, name of port 2.The connecting line is an auxiliary element which is connected with the power device, which can automatically generate the connection relationship in the process of connecting the device components. Then draw the corresponding network wiring diagram by the above graphic elements according to the actual hydropower plant electrical wiring structure.

The merging procedures of the connecting line ports are as follows: (1) Number all the ports of the electrical components in the network automatically, being 1,2,3 ..12 successively as shown in Fig. 3; (2) Name the numbers of the component ports connecting to the two ends of the connecting line as P1 and $\mathrm{P} 2$ respectively. If $\mathrm{P} 1$ is equal to $\mathrm{P} 2$, no treatment is conducted; ( 3 ) If $\mathrm{P} 1$ is not equal to $\mathrm{P} 2$, omit the bigger number. The specific practice is that if $\mathrm{P} 1>\mathrm{P} 2$, then $\mathrm{P} 1=\mathrm{P} 2$, else $\mathrm{P} 2=\mathrm{P} 1$; (4) Compared with all the ports of the components in the network, if the component port number P1 or P2 is bigger than the bigger number in Procedure 3, the port number minus 1. After the "merging of the connecting line" stated above, the physical port numbers of the main connection diagram in Fig. 3 are shown in Fig. 4. The connection between the electric power equipment can be described by the program language as follows: transformer[1]. node2= breaker[1].node1.

In the practical application, it is needed sometimes to judge the interlock relationship between the equipment except for the switch. For the universal modeling, expand the conception of the interlock with the view that the interlock includes the interlock relationship among the bus, the transformer and the lines as well. From Fig. 4, it is clearly shown that the two ports of the circuit breaker have the same physical numbers with one port of the isolating switches on the two sides respectively. It can be determined that the equipment requiring the judgment of interlock state is the isolating switches 50111 and 50112 in the operation process of the circuit breaker 5011 through the same physical numbers. Take the process of the "operating state" transferring to "hot stand-by state" of the circuit breaker 5011 as the example. There is the logical interlock function $F\left(K_{5011}\right)=K_{50111} * K_{50112}$. That is, when $K_{5011}$ is operated, $K_{50111}$ and $K_{50112}$ shall be under switching on state.

The topology analysis algorithm based on the "merging of the connecting line" can create the information table of the interlock relationship automatically. After the change of the main electrical connection, just modify the connection diagram and re-operate the topology analysis program. And the interlock relationship information table can form automatically again, strengthening the generality of all the electrical switch models to make the models adapt to the different electrical switch system in the hydropower plants.

Simulation Example. The modular and graphical modeling approach of the electrical switch system stated above was applied in the simulation training system project of Longtan Hydropower plant. This station has 9 synchronous generators with the installed capacity being $700 \mathrm{MW}$ which are connected to the duplex-wound transformer by the unit connection way through the outlet of the generators and in turn connected to the $500 \mathrm{Kv}$ switch station through the high-voltage cable. The main connection of this $500 \mathrm{kV}$ GIS switch station adopts double bus $4 / 3$ connection way in 5 strings totally with 6 outgoing lines. The interface of switch simulation system is shown in Fig. 5. 


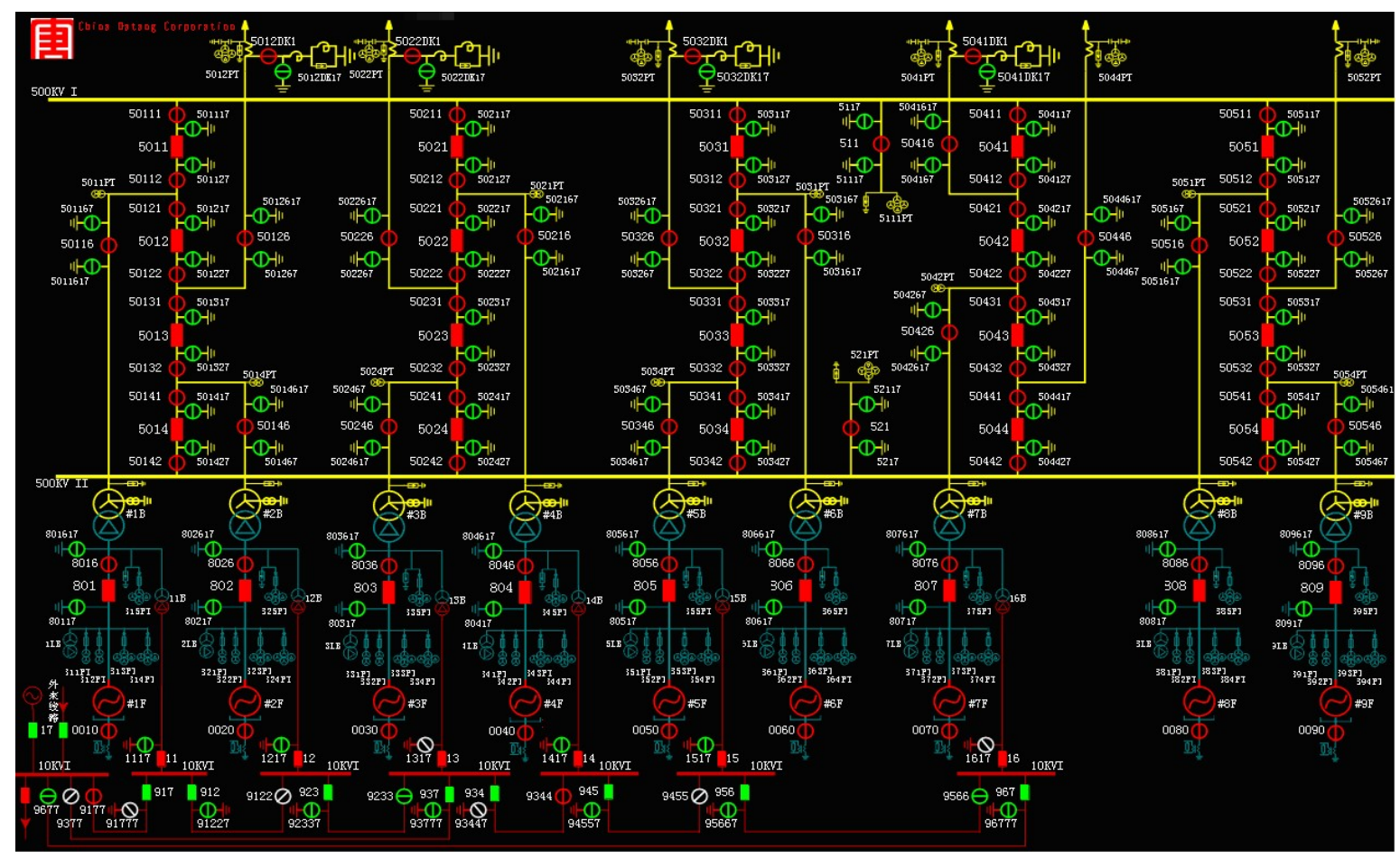

Figure 5. hydropower plant switch simulation system

\section{Summary}

The mass customization problem of the hydropower plant simulation system has not been solved well. The key to this problem is how to make the simulation model universal and adaptive. The hierarchy modular modeling approach is adopted to layer and classify all the switches of the hydropower plant. It is easy to set up universal enough simulation model for the sub-modules and then establish the complete switch system with the modular combination by the graphical method. A new method is proposed to judge the logic interlock in the course of switching action using the logic interlock function. The topology analysis algorithm based on the "merging of the connecting line" can create the information table of the interlock relationship automatically. The hydropower switch simulation system is designed and developed on the basis of this method. The practical verification of the simulation system in large-scale hydropower plants indicates that, the method solve the problems of model development, operating speed and model extendibility, and accordingly provide an effective scheme for the mass customization of hydropower plant simulation system.

\section{References}

[1] Chengming WU, Xianshan LI, Binqiao ZHANG, Modularization Modeling for Real Time Simulation of Hydropower Plants, The 2008 International Conference on Electrical Engineering , Hong Kong, 2008.3.19-3.21.

[2] Feng Zhu, Yiping Yao, Wenjie Tang, A high performance framework for modeling and simulation of large-scale complex systems[J]. Future Generation Computer Systems. 51 (2015) 132-141.

[3] Yin Guiliang,Zhang Shengming, Modeling and simulation of micro-hydropower unit system[J]. Power System Technology. 36 (2012) 147-152

[4] Chen Wang, Qingyun Duan,Charles H.Tong,Zhenhua Di, Wei Gong, A GUI platform for uncertainty quantification of complex dynamical models[J]. Environ. Model. Softw. 76 (2016) 1-12

[5] Juan Garrido, Ángel Zafra, Object oriented modelling and simulation of hydropower plants withrun-of-river scheme[J]. Simulation Modeling Practice and Theory. 16 (2009) 1748-1767 